\title{
Foundations for Fitness: Initial Outcomes from a 10-Week Multidisciplinary, Pediatric Obesity Intervention
}

\author{
Steven Smith ${ }^{2 *}$, Kyle M. Morrison ${ }^{1}$, Jessica Krantz ${ }^{2}$ and Jorgie Watson ${ }^{2}$ \\ ${ }^{1}$ Department of Kinesiology, Hope College Kinesiology, USA \\ ${ }^{2}$ Director of the Foundations for Fitness program, Hope College Kinesiology, USA \\ ${ }^{*}$ Corresponding author: Steven Smith, PhD, Hope College Kinesiology, Foundation for Fitness, 222 Fairbanks Ave. Holland \\ Submission: 侮 October 27, 2017; Published: 望 January 25, 2018
}

\begin{abstract}
Childhood overweight and obesity is a national epidemic and predisposes children to adulthood obesity as well as increases the risk of developing cardiovascular, metabolic, and psychological disorders. Prior research has found that if an individual can engage in lifestyle modification for 10 weeks then the change is more likely to be maintained after the intervention. The purpose of this study was to assess the effectiveness of a 10 week multidisciplinary intervention program on improving the health outcomes of children aged 7-12 years with a body mass index (BMI) greater than the $85^{\text {th }}$ percentile. Child participants were referred from local pediatric care providers and, of the 15 that began the program, 13 participants completed the 10-week training session. Subjects were assessed for resting heart rate, resting blood pressure, BMI, percent body fat, waist circumference, height and weight. Furthermore, participants completed a quality of life survey and the participating parents filled out a questionnaire assessing the obesogenic factors in the household (FNPA). Prior to pre-program evaluations, the parents of participants completed an informed consent form approved by a Human Subjects Review Board. All children participated in the exercise portion of the program and each training session consisted of an hour and a half of physical activity once per week led by physical education students. Parents of participants simultaneously attended educational sessions on lifestyle modification. At the end of the program waist circumference significantly decreased ( $\mathrm{p}=.027$ ) and scores for the FNPA were trending towards significant improvement. Data collection from the second cohort of the intervention program is currently ongoing.
\end{abstract}

\section{Introduction}

It is clear in the research literature and from anecdotal observation that the obesity epidemic affecting our world is not abating. The continuing impact of this problem is evident in many areas of our society from healthcare cost to social emotional cost to the individual. It is a far-reaching epidemic that professionals have sought to curb and turn back for the sake of a generation of children and adults. It appears that most programs that have sought to impact this problem have been marginally successful at best and a failure at worst. A review of the literature reveals that while the problem has grown our intervention approaches have been like the proverbial finger in the dike to stop the water from breaching the dam and overflowing into our culture.

The Center for Disease Control (CDC) website has summarized that "childhood obesity has more than doubled in children and quadrupled in adolescents in the past 30 years" [1-3]. Additionally, the CDC reports that $17 \%$ (12.7 million) children ages 2-19 years are obese! With such devastating trends there have been many programs seeking to impact the impact of childhood obesity in the United States and indeed across the world. Most have reported only marginal success or no impact at all on curbing the trend. Finding a scientifically based successful intervention has proven to be very difficult.

\section{Background}

It is clear that there are many factors contributing to the rise in childhood obesity. Physical inactivity, caloric imbalance, built environments, psychosocial variables and family dynamics all seem to contribute to the current problems. Some of these problems include fatty liver disease, obstructive sleep apnea, diabetes and other orthopedic problems. Even obese children who did not have reported physical conditions still claimed low quality of life [4]. The co-morbidities associated with obesity are completely preventable yet are the number one cause of preventable deaths in the United States [2].

As the prevalence of obesity in United States adolescents approaches $20 \%$, there is an increased need to determine effective interventions that produce long-term results. In his discussion on weight loss strategies for adolescents, Ludwig emphasized the influence parents have on weight-related behavior [5]. With an increase in childhood obesity, secondary complications are 
diagnosed with greater frequency such as Type 2 diabetes, hypertension, fatty liver disease, and sleep apnea. Furthermore, overweight and obese children have a greater risk of developing depression [6]. Addressing the growing childhood obesity epidemic from a multidisciplinary approach which focuses on reducing the obesogenic factors in the home environment has proven an effective method in reducing the prevalence of obesity [7].

\section{Objective}

The objective of the program entitled Foundation for Fitness is to improve children's health and fitness through physical activity, motor skill development, parental support, and education about community resources for healthy living. This particular study sought to discover whether the initial interventions in the program had any effect on childhood obesity from a multidisciplinary perspective. The specific purpose of this study was to assess the effectiveness of a 10 week multidisciplinary intervention program on improving the health outcomes of children aged 7-12 years with a body mass index (BMI) greater than the 85th percentile.

\section{Methods}

Child participants were referred from local pediatric care providers and had a BMI of greater 85 th percentile ranking. Of the 15 children ranging in age from 7-12 years that began the program, 13 participants completed the 10 -week training session. Subjects were assessed for resting heart rate, resting blood pressure, BMI, percent body fat, waist circumference, height and weight. Furthermore, participants completed a quality of life survey and the participating parents filled out a questionnaire assessing the obesogenic factors in the household (FNPA). Prior to pre-program evaluations, the parents of participants completed an informed consent form approved by a Human Subjects Review Board.

All children participated in the exercise portion of the program and each training session consisted of an hour and a half of physical activity once per week led by physical education students from Hope College in Holland, Michigan. The activities included such game-like activities as kickball, tagging games, swimming, and modified capture the flag. Other sessions included "commercial break" activities whereby children participated in short activities that they could use while a commercial would come on their television break (average TV break is four minutes). Additionally, the children participated weekly in a strength circuit and an iStep program (using a computer based dance program). An exercise gaming stationary bike was also used on a weekly basis. The pedalling powers the play, and participants race each other in Pixar's Cars video game where the controller is powered by the subject pedalling. Each child was also matched with a college mentor that would perform physical activity with the child for 90 minutes per week.

While the children participated in physical activity, the parents of participants simultaneously attended educational sessions on lifestyle modification, presentations from dieticians, and mental health specialists skilled at presentations on peer relationships, self-esteem and physical activity. In addition to the cognitive and practical sessions, a gymnasium membership to the college facilities was also included for the duration of the program giving the entire family access to the exercise equipment in addition to the weekly sessions of the Foundation for Fitness.

\section{Results}

Subjects were assessed for resting heart rate, resting blood pressure, BMI, percent body fat, waist circumference, height and weight. Furthermore, participants completed a quality of life survey and the participating parents completed a questionnaire assessing the obesogenic factors in the household (FNPA). The FNPA showed a trend toward positive significance. This FNPA trending towards significance is important to analyze as it predicts whether the changes observed will be long term in nature. It also shows the motivation of parents to change their environment and what they have learned from attending the lecture portion of the program.

At the end of the program waist circumference (WC) significantly decreased $(p=.027)$ (Figure 1) and scores for the obesogenic factors in the household (FNPA) were trending towards significant improvement (Figure 2). Participants reported a reduction of one pant size on average per pupil. Subjects showed minimal increases in BMI and minimal increases in percent of body fat as well. Slight decreases in systolic blood pressure were reported as well.
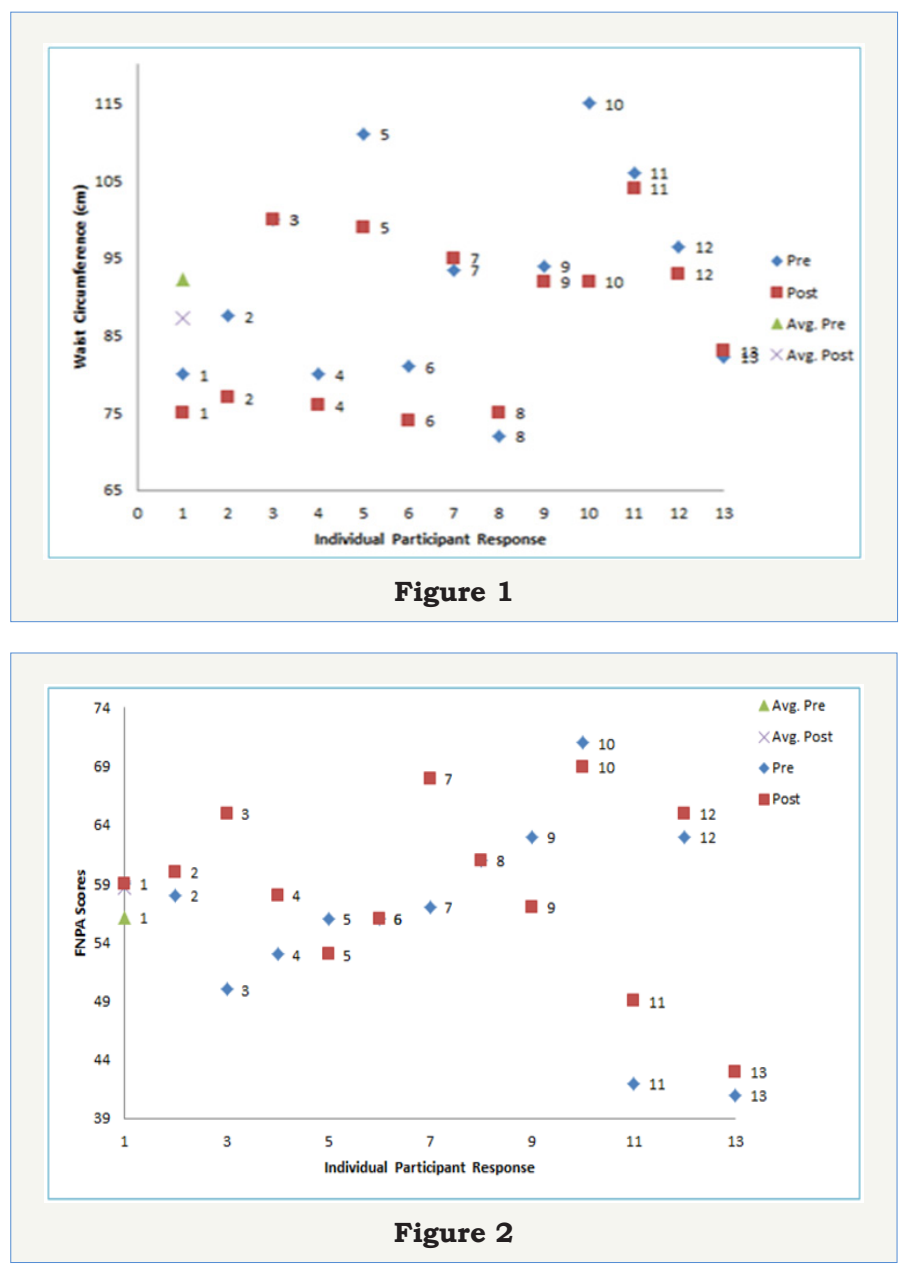


\section{Conclusions and Discussion}

There are some limitations to this particular study as the sample size is quite small to determine significance and generalize findings to a larger population. It is possible that trends may have been more significant with a larger pool of data which would improve the beta. Other limitations include the testing date having been scheduled for the week after Thanksgiving. This usually means heavy salt consumption from traditional holiday food which can mean greater water retention and increased weight and BMI for final testing. Further cohorts to test the effectiveness of the program are currently underway.

The author's suggestions for further research include:

- $\quad$ Continue community engagement.

- Investigation into relationships between motor development and weight status.

- $\quad$ Physical Activity Assessment through questionnaires.

- Continue collecting data on subsequent co-horts .
- $\quad$ Modify program based upon feedback from participating families \& student volunteers.

\section{References}

1. Ogden CL, Carroll MD, Kit BK, Flegal KM (2014) Prevalence of childhood and adult obesity in the United States, 2011-2012. Journal of the American Medical Association 311(8): 806-814.

2. National Center for Health Statistics (2012) Health, united states, 2011: with special features on socioeconomic status and health. US Department of Health and Human Services.

3. Childhood Obesity Facts.

4. Schwinner, Burwinkle TM, Varni JW (2002) Health related quality of life of severely obese children and adolescents. JAMA 289(14): 1813-1819.

5. Ludwig D (2012) Weight loss strategies for adolescents: A 14-year-old struggling to lose weight. JAMA 307(5): 498-508.

6. Eneli I, Cunningham A, Woolford S (2008) The pediatric multidisciplinary obesity program: An update. Progress in Pediatric Cardiology 25(2): 129136.

7. Spear B, Barlow S, Ervin, C, Ludwig D, Saelens B, et al. (2010) Recommendations for treatment of child and adolescent overweight and obesity. Pediatrics 120: S254-S288. 